\title{
Adaptive resource allocation for SDMA-OFDMA systems with genetic algorithm
}

\author{
Berna Özbek \#, Didier Le Ruyet * \\ \# Electrical and Electronics Engineering Department, Izmir Institute of Technology \\ Urla, Izmir, 35430, Turkey \\ bernaozbek@iyte.edu.tr \\ * Electronics and Communications Laboratory, Conservatoire National des Arts et Métiers (CNAM) \\ 292 rue Saint Martin, 75141, Paris, France \\ didier.le_ruyet@cnam.fr
}

\begin{abstract}
In this paper, we propose an adaptive resource allocation algorithm to maximize the sum data rate for spatial division multiplexing access (SDMA) orthogonal division multiple access (OFDMA) systems using genetic algorithm. We perform Zero forcing $(\mathrm{ZF})$ beamforming at the transmitter constructing single and multiple beams for OFDMA systems with multiple transmit antennas. In order to reduce the complexity and convergence time of genetic algorithm (GA), we present an initial condition obtained by allocating the user to the subcarrier with maximum precoding gain. The proposed algorithm reduces the complexity without sacrificing the performance. We obtain the simulation results and compare them with the classical static and adaptive allocation methods. We show that the GA with the proposed initial condition increases the sum data rate compared to classical GA and reduces the convergence time significantly for SDMA-OFDMA systems. ${ }^{1}$
\end{abstract}

\section{INTRODUCTION}

For next generation wireless communication systems, the wireless systems in the bandlimited channels and with intersymbol interference (ISI) could support the high data rate broadband applications such as wireless internet access, wireless multimedia and future mobile systems. For these applications, orthogonal frequency division multiplexing (OFDM) that is already involved in many wireless communication standards is one of the promising modulation technique because of its ability to combat ISI over multipath fading channels. Combining OFDM systems with multiple antenna techniques, the performance of communication systems in frequency selective channels improves significantly.

For single user OFDM systems, the Greedy algorithm (adaptive bit and subcarrier allocation) gives the optimal solution to minimize the overall power allocation when the channel coefficients of each subcarrier are known at the transmitter. However, for the multiuser OFDM systems, it is much more difficult to find the optimal solution since a subcarrier allocated to a user can also be the the best for other users.

The subcarrier and bit allocation for OFDMA systems can be separated into static and dynamic allocation. The static subcarrier allocation schemes use fixed resource allocation schemes namely time division multiple access division

\footnotetext{
${ }^{1}$ The work was partially supported by the Tubitak-PIA Bosphorus Project.
}

(TDMA) and frequency division multiple access (FDMA) [1]. In these schemes, the channel state information is ignored and each user is allocated for a predefined time slot or frequency band.

For dynamic subcarrier and bit allocation algorithms, it is assumed that the channel coefficient for each subcarrier is available at the transmitter. In [2], iterative searching algorithm applying Lagrangian relaxation for optimum multiuser bit, subcarrier and power is proposed. This algorithm gives a solution close to the optimum solution but the computation complexity is high. In order to simplify the allocation problem, each subcarrier is allocated to the user that has the maximum channel gain for this subcarrier [3]. This algorithm is very simple but cannot achieve all the multiuser diversity gain. A low complexity solution for the problem described in [2] is presented in [4] and it is shown that the performance results approaches the optimum solution. In [5], a resource allocation solution that reaches the optimum solution with reduced complexity to maximize sum data rate is described by considering capacity calculations. Furthermore, a subcarrier and bit allocation by using genetic algorithm (GA) is presented in [6] and [7] for the problem of the total transmit power minimization with fixed rate constraints . In order to reduce to convergence time of GA, an initial condition is proposed in [6] considering efficiency (when allocating, the users that have the highest channel gain are preferred since they demand less power) and fairness (a number of subcarriers are allocated to each user without considering their channel gains) criteria. In [7], the GA parameters are adapted during the iteration process to reduce the convergence time.

For OFDMA systems with multiple transmit antennas, the resource allocation includes precoding vector selection, subcarrier allocation and bit loading [8]. The Space Division Multiple Access (SDMA) uses a beamforming technique in a multiple-antenna system and multiplexes multiple users on the same subcarrier to increase the spectral efficiency.

In this paper, we propose to allocate precoding vector, subcarrier and bit/power by using GA to maximize sum data rate for OFDMA systems with multiple transmit antennas. In this scheme, we perform Zero forcing $(\mathrm{ZF})$ beamforming at the 
transmitter constructing single and multiple beams. In order to reduce the complexity and convergence time of GA, we propose an initial condition which is obtained by allocating the user to the subcarrier having maximum precoding gain.

First, we describe the system model of SDMA-OFDMA systems over wireless channels in section 2 . Then, we present the adaptive resource allocation algorithm for describing GA in section 3. Finally, in section 4, we present the simulation results by evaluating the sum data rate and the convergence time.

\section{SYSTEM MODEL FOR SDMA-OFDMA}

We consider a SDMA-OFDMA system with $N_{t}$ transmit antennas, $K$ users with single antenna and $N$ subcarriers as drawn in Figure 1. In this model, the channel vector between the base station and the $k$ th user for the $n$th subcarrier is described by

$$
\mathbf{H}_{k, n}=\left[\begin{array}{llll}
H_{k, n, 1} & H_{k, n, 2} & \ldots & H_{k, n, N_{t}}
\end{array}\right]
$$

where $H_{k, n, t}$ is the channel information from the $t$ th transmit antenna to the $k$ th user for the $n$th subcarrier.

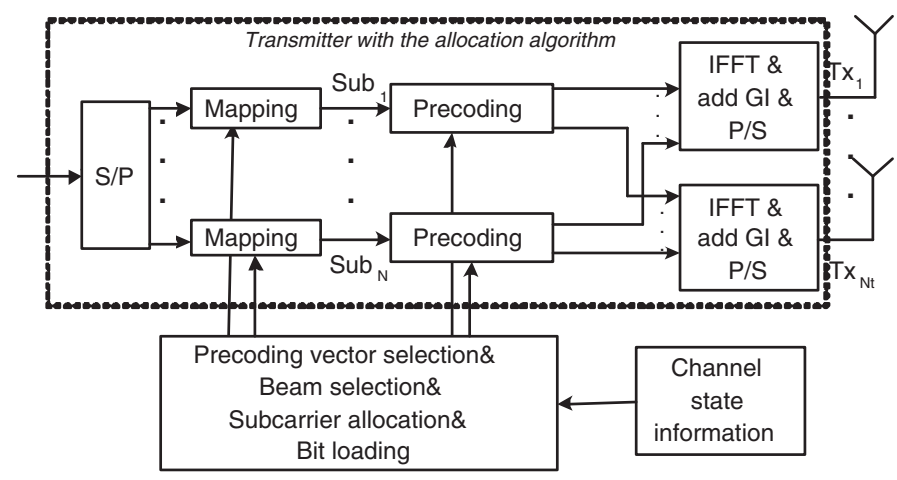

Fig. 1. The transmitter structure for OFDMA system with multiple transmit antennas.

For a system where $N_{t} \leq K$, let $\mathcal{S}_{n}$ be the set of $N_{t}$ users scheduled at subcarrier $n$ and the associated users' data are transmitted using ZF precoding. Then $\mathbf{H}\left(\mathcal{S}_{n}\right)$ denotes the matrix consisting of $N_{t}$ channel vectors of the selected users at subcarrier $n$. The relation between the data vector $\mathbf{S}_{n}\left(\mathcal{S}_{n}\right)$ and the received signal for $k$ th user and $n$th subcarrier can be written as:

$$
\begin{aligned}
Y_{k, n} & =\mathbf{H}_{k, n} \mathbf{X}\left(\mathcal{S}_{n}\right)+N_{k, n} \\
& =\mathbf{H}_{k, n} \mathbf{W}\left(\mathcal{S}_{n}\right) \mathbf{P S}\left(\mathcal{S}_{n}\right)+N_{k, n}
\end{aligned}
$$

where $\mathbf{P}$ is a diagonal matrix consists of power allocation between the subcarriers and the beams, $\mathbf{X}\left(\mathcal{S}_{n}\right) \in \mathcal{C}^{N_{t} \times 1}$ is the transmitted symbol from the base station antennas.

The ZF transmit beamforming matrix is given by

$$
\mathbf{W}\left(\mathcal{S}_{n}\right)=\alpha \mathbf{H}\left(\mathcal{S}_{n}\right)^{H}\left(\mathbf{H}\left(\mathcal{S}_{n}\right)^{H} \mathbf{H}\left(\mathcal{S}_{n}\right)\right)^{-1}
$$

In order to keep the short term power constant, we have:

$$
\alpha=\frac{1}{\sqrt{\operatorname{tr}\left(\left(\mathbf{H}\left(\mathcal{S}_{n}\right)^{H} \mathbf{H}\left(\mathcal{S}_{n}\right)\right)^{-1}\right)}}
$$

The precoding matrix, $\mathbf{W}\left(\mathcal{S}_{n}\right)$ is defined by,

$$
\mathbf{W}\left(\mathcal{S}_{n}\right)=\left[\begin{array}{llll}
\mathbf{W}_{1}\left(\mathcal{S}_{n}\right) & \mathbf{W}_{2}\left(\mathcal{S}_{n}\right) & \ldots & \mathbf{W}_{N_{t}}\left(\mathcal{S}_{n}\right)
\end{array}\right]
$$

where $\mathbf{W}_{b}\left(\mathcal{S}_{n}\right)$ is the precoding vector for the $b$ th beam and the $n$th subcarrier with the dimension of $N_{t} \times 1$. In order to maximize the sum data rate, the optimization problem can be given as

$$
\max _{\mathbf{W}_{n} ; c_{n} ; \rho_{n}} R=\max _{\mathbf{W}_{n, b} ; c_{n, b} ; \rho_{n, b}} \sum_{b=1}^{N_{t}} \sum_{k=1}^{K} \sum_{n=1}^{N} c_{n, b} \rho_{k, n, b}
$$

subject to

$$
\sum_{b=1}^{N_{t}} \sum_{k=1}^{K} \sum_{n=1}^{N}\left(\frac{f\left(c_{n, b}\right) \rho_{k, n, b}}{\left|\mathbf{H}_{k, n} \mathbf{W}_{b}\left(S_{n}\right)\right|^{2}}\right) \leq P_{T}
$$

and

$$
\sum_{k=1}^{K} \rho_{k, n, b}=1 \text { for each } n, b
$$

where $P_{T}$ is the total transmit power and

$\rho_{k, n, b}= \begin{cases}1 & k \text { th user is allocated to the beam } b \text { for subcarrier } n \\ 0 & \text { else }\end{cases}$ Consequently, only one user is allocated to each subcarrier and each beam.

For M-ary quadrature amplitude modulation (M-QAM) schemes, the bit error probability is upper bounded by the symbol error probability, which is tightly approximated by $4 Q\left(\sqrt{d^{2} /\left(2 N_{0}\right)}\right)$ [9] where $d$ is the minimum distance between the points in the signal constellation. Since the average energy of a M-QAM symbol is equal to $(M-1) d^{2} / 6$, then the required power (in energy per symbol) $f\left(c_{n, b}\right)$ for supporting $c_{n, b}$ bits per symbol at a required bit-error-rate (BER) $p_{e}$ can be represented by

$$
f\left(c_{n, b}\right)=\frac{N_{0}}{3}\left[Q^{-1}\left(p_{e} / 4\right)\right]^{2}\left(2^{c_{n, b}}-1\right)
$$

where $N_{0} / 2$ denotes the variance of the additive white Gaussian noise (AWGN) and $Q(x)=\frac{1}{\sqrt{2 \pi}} \int_{x}^{\infty} \mathrm{e}^{-t^{2} / 2} d t$.

\section{AdAptive Resource Allocation With GenetiC Algorithm}

GA is based on the mechanism of natural selection of stronger individuals. GA is an attractive algorithm for some types of optimizations by improving the computational performance [6]. In this work, we propose to use the GA to optimize the precoding, subcarrier and bit allocation for SDMA-OFDMA systems to maximize the sum data rate. This optimization problem is a difficult non-convex optimization problem. In this algorithm, each subcarrier and each OFDM symbol is modeled as a gene and chromosome respectively. The number of genes in a chromosome is equal to the number of multiplication of subcarriers and number of transmit antennas in an OFDM symbol. The value of each gene is confined to the integer from 1 to $K$. For example, if the first 
gene of the chromosome is $k$ for beam $b$, then the subcarrier is allocated to the user $k$ for beam $b$.

The algorithm is described as follows:

\section{Step 1)Initial population:}

It includes $W$ chromosomes.

\section{Step 2)Evaluate:}

Fitness function is chosen as the sum data rate. The higher the sum data rate, the better is the fitness of the chromosome. For each chromosome, after applying bit allocation to the given subcarrier and beam allocation, the sum data rate is calculated using (7) considering the constraints defined in (8) and (9). The precoding vector is determined using the ZF beamforming.

Step 3)Generate the new population:

Selection: The $P$ chromosomes that have the lowest fitness are discarded. The remaining $W-P$ chromosomes are applied to the crossover and mutation processes and are then selected for the new population. The best $P$ chromosomes are directly added to the new population without applying any process.

Crossover: The probability of crossover is set to $P_{c}$ and $(W-P) * P_{c}$ chromosomes are applied to the two-point crossover and then passed to the new population. The remaining $(W-P) *\left(1-P_{c}\right)$ chromosomes are directly passed to the new population.

Mutation: The probability of mutation is set to $P_{m}$ and after the crossover process $(W-P) * P_{m}$ chromosomes are applied to the mutation and added to the new population. The remaining $(W-P) *\left(1-P_{m}\right)$ chromosomes are directly added to the new population without any mutation. In order to avoid the convergence of the algorithm to a local maximum solution, the probability of mutation is changed to $P_{m 1}$ when no better individuals is found in 5 consecutive generations, to $P_{m 2}$ when no better individuals is found in 10 consecutive generations and to $P_{m 3}$ when no better individuals is found in 15 consecutive generations. $G_{m}$ among the $\mathrm{N}$ gene of the selected chromosomes are applied to the mutation.

\section{Step 4)}

Repeat the Step 2-4 until the predefined generation number (assumed to be $D$ ) is reached or no better array is found after 20 consecutive generations. The best array in the population is the final solution.

\section{A. The Proposed Algorithm}

The reduction of the number of iterations to reach the final solution is one of the important issue in the GA in order to reduce the convergence time and the complexity of the algorithm. For this purpose, the parameters should be determined properly and the initial population should include the good chromosomes to accelerate the process. In the classical GA, the initial population is generated randomly. In [6] and [7], for multiuser OFDM systems, a chromosome that minimizes the transmit power is added to the initial population in order to reduce the number of iterations. In this paper, we propose to reduce the number of iteration and consequently the convergence time by adding a special chromosome that maximize the sum data rate to the initial population.
Modified Step 1) While reconstructing the initial population, $W-1$ chromosomes are generated randomly as in classical GA and the last chromosome is obtained by allocating the subcarriers to the beam and the user with the maximum precoding gain.

For each subcarrier, the user index that has maximum precoding gain is selected for each beam as,

$$
\kappa_{n}, \beta_{n}=\max _{k, b}\left|\mathbf{H}_{k, n} \mathbf{W}_{b}\left(S_{n}\right)\right|^{2}
$$

where $n=1,2, \ldots, N, b=1, \ldots, N_{t}$.

Then, for each subcarrier and each beam, the users are allocated as,

$$
\rho_{\kappa_{n}, n, \beta_{n}}=1
$$

\section{Simulation Results}

In this section, considering the sum data rate performance, we compare the proposed GA with with TDMA, FDMA, maximum user selection and the classical GA algorithms. Furthermore, we obtain the performance results of convergence time for GA with modified Step 1 and classical GA.

We perform the simulation results using a typical office environment with 9 channel taps. The total OFDM symbol duration is $4 \mu \mathrm{s}$ including $0.8 \mu \mathrm{s}$ guard interval. It consists of $N=64$ subcarriers. The Doppler frequency is set to $50 \mathrm{~Hz}$. The required BER and the noise variance are chosen as $p_{e}=10^{-3}$ and $N_{0}=1$ respectively. The number of bits per symbol is selected as $M=0,1,2,4,6$ that corresponds to modulation types BPSK, QPSK, 16QAM, 64QAM. The transmitted power is fixed at $P_{T}=20 \mathrm{dBW}$. The average data rates are calculated for one OFDM symbol to obtain results independent from the frame length. The number of transmit antennas is chosen as $N_{t}=2$.

The parameters for GA is listed in Table I as following:

TABLE I

PARAmeters For Genetic Algorithm

\begin{tabular}{|c|c|}
\hline Parameters & Values \\
\hline$W$ & 36 \\
\hline$P$ & 6 \\
\hline$P_{c}$ & 0.8 \\
\hline$P_{m}$ & 0.1 \\
\hline$P_{m 1}$ & 0.2 \\
\hline$P_{m 2}$ & 0.4 \\
\hline$P_{m 3}$ & 0.5 \\
\hline$G_{m}$ & 2 \\
\hline$D$ & 200 \\
\hline
\end{tabular}

For TDMA, each user is assigned a predetermined time slot and can use all the subcarriers within that time slot. For FDMA, each user is assigned a predetermined band of subcarriers and can only use those subcarriers in every OFDM symbol. In order to compare static allocation techniques to dynamic resource allocation techniques fairly, the bit loading is also applied to TDMA and FDMA. The maximum user selection algorithm is performed by exhaustive search considering all combination of the user pairs for beams for each subcarrier. 
In Figure 2, the sum data rate values is shown for the classical and proposed GA at each iteration for $K=4$ and 8 users respectively constructing only one beam. It is observed we obtain a lower average convergence time for the proposed GA than the classical one by only adding one good chromosome to the initial population.

For different users, the average iteration number is drawn in Figure 3 and it is shown that the proposed algorithm has a better convergence performance than the classical one.

In Figure 4, the sum data rate results with one beam OFDMA systems are compared to existing algorithms and it is shown that the proposed algorithm gives the best performance.

In Figure 5, according to the results for two beams, the better sum data rate results are obtained compared to classical one.

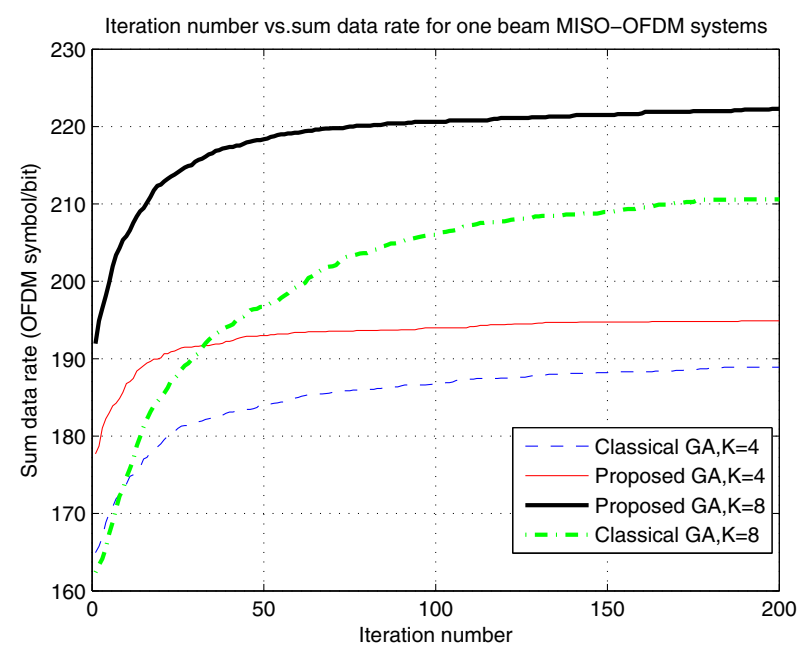

Fig. 2. Iteration number versus sum data rate for $K=4$ and 8 for one beam OFDMA systems.

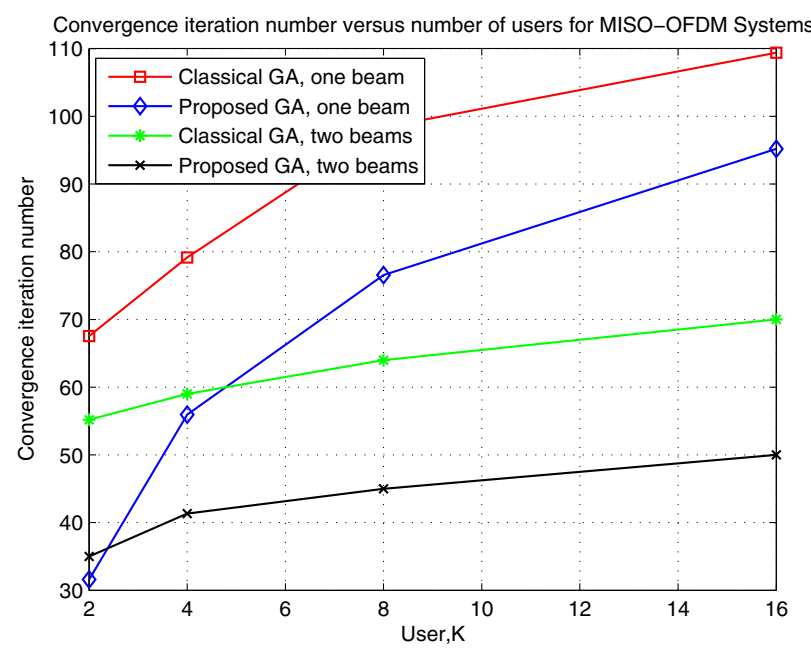

Fig. 3. Convergence iteration versus number of users for SDMA-OFDMA systems.

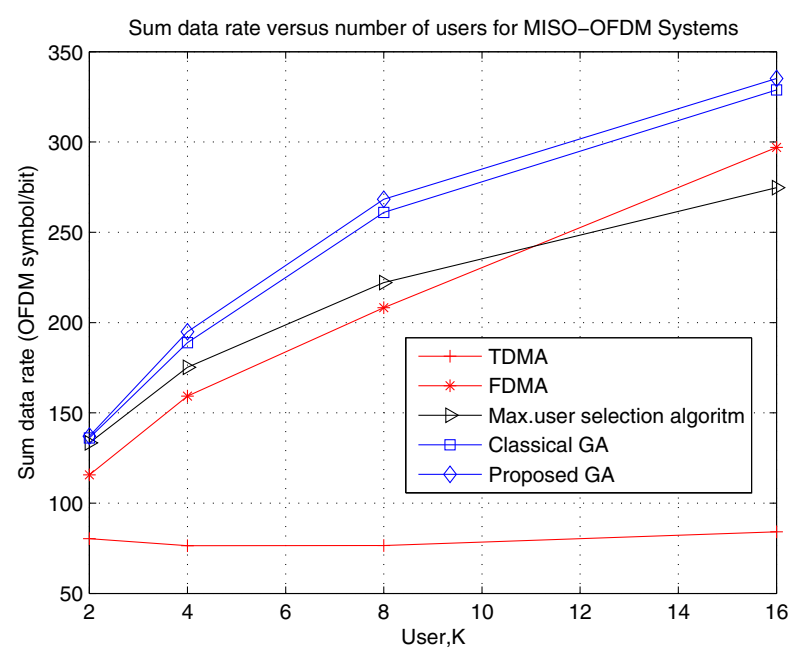

Fig. 4. Average sum data rate versus number of users for OFDMA systems with one beam.

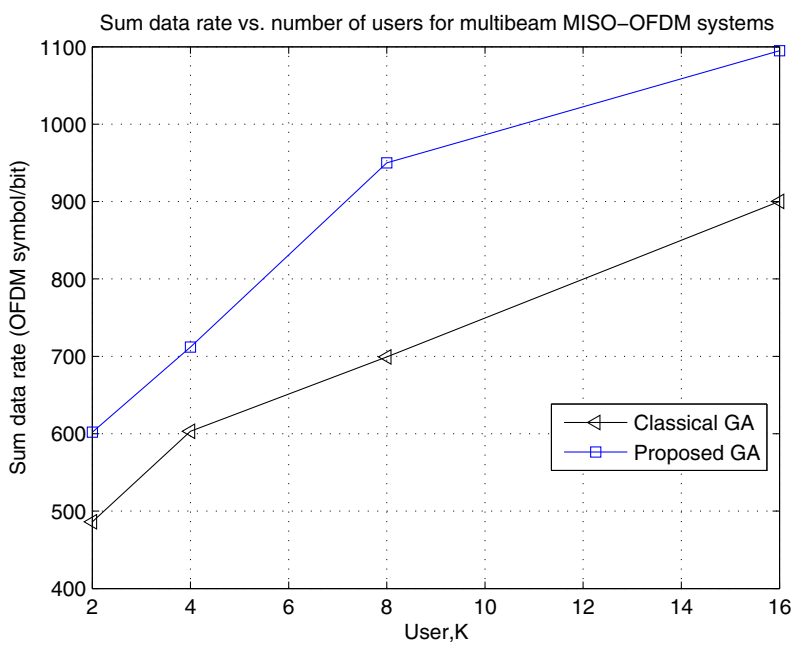

Fig. 5. Average sum data rate versus number of users for SDMA-OFDMA systems with two beams.

\section{CONCLUSION}

In this paper, we have proposed a resource allocation for SDMA-OFDMA systems with ZF beamforming by using the genetic algorithm for the multiple antennas case. The aim was to maximize the sum data rate however the proposed algorithm can also be used to minimize the transmit power. The proposed algorithm allocates more than one beam when it increases the sum data rate. Furthermore, in order to accelerate the convergence time of the GA, we have added a good chromosome to the initial population. This chromosome is obtained by allocating users to the beams with a maximum precoding gain. The performance results shown that using two beams increase the sum data rate while the required convergence time is reduced significantly with proposed solution. 


\section{REFERENCES}

[1] R. Prasad, OFDM Wireless Multimedia Communications. Artech House, 2000.

[2] C. Y. Wong, R. Cheng, K. B. Letaif, and R. D. Murch, "Multiuser ofdm with adaptive subcarrier, bit and power allocation," IEEE J. Sel. Areas Commun., vol. 17, pp. 1747-1758, 1999.

[3] E. Bakhtiari and B. Khalaj, "A new joint power and subcarrier allocation scheme for multiuser ofdm systems," in Proc. IEEE PIMRC'03, 2003, pp. 1959-1963.

[4] G. Zhang, "Subcarrier and bit allocation for real-time services in multiuser ofdm services," in Proc. ICC'04, 2004, pp. 2985-2989.

[5] W. Rhee and M. J. Cioffi, "Increase in capacity of multiuser ofdm system using dynamic subchannel allocation," in Proc. VTC'00, 2000, pp. 10851089.

[6] Y. Wang, F. Chen, and G. Wei, "Adaptive subcarrier and bit allocation for multiuser ofdm system based on genetic algorithm," in Proc. ICCCAS'05, 2005, pp. 242-246.

[7] Y. Reedy and N. Gajendar, "Evolutionary approach for efficient resource allocation in multiuser ofdm systems," Journal of Communications, vol. 2, pp. 42-48, 2007.

[8] Y. J. Zhang and K. Letaief, "An efficient resource-allocation scheme for spatial multiuser access in mimo/ofdm systems," IEEE Trans. Commun., vol. 53, pp. 107-116, 2005.

[9] J. G. Proakis, Digital Communication. New York:McGrawHill, 1995. 